\title{
Light and current generation system for measuring the behaviour of the Norway lobster
}

\author{
David Sarriá ${ }^{*}$, Valerio Sbragaglia ${ }^{2}$, Spartacus Gomáriz ${ }^{1}$, José Antonio \\ García $^{2}$, Carla Artero ${ }^{1}$, Jacopo Aguzzi ${ }^{2}$, Francesc Sardà ${ }^{2}$, Antoni Mànuel ${ }^{1}$ \\ 1 SARTI Research Group. Department of Electronic Engineering. Universitat \\ Politècnica de Catalunya. Rambla de l'Exposició 24, 08800 Vilanova i la Geltrú, Spain.
} Email: david.sarria@upc.edu Tel: +34938967200

2 Marine Science Institute, (ICM-CSIC) - Passeig Marítim de la Barceloneta 37-49, 08003 Barcelona, Spain.

Patterns of how the Norway lobster (Nephrops norvegicus L.) emerges from its burrow in relation to sea currents at different times of the day-night cycles is currently unknown. The degree of influence of these variables when simulated in controlled laboratory conditions entails great technical and technological difficulties. This paper presents the development of an integrated control system that manages a set of aquariums generating water currents and patterns of light. The solution proposed combines the use of commercial equipment and a custom electronic design in order to achieve the required specifications with costminimization. The control system has circulation pumps and visible and infrared LED lighting to recreate different currents and light conditions. Preliminary results show the control system works properly and all components responded as expected during the experiments.

Keywords: Wireless control, hydrodynamic aquarium regulation, lighting control, variable frequency drive, microcontroller

\section{Highlights:}

- The electronic system implemented allows the study of marine species chronobiology.

- The system consists of a custom electronic board, circulation pumps and LED lighting. 
- The configurable water flow and light conditions recreate different sea bed scenarios.

- Wireless interfaces reduce cost and installation complexity in aquariums.

- The infrared lighting enables the study of species in the dark with cameras.

\section{Introduction}

Marine habitat degradation, pollution and especially overfishing are the main reasons why 76 per cent of world fish stocks dials are overexploited or depleted and at risk of extinction if they are not managed properly (Csirke, 2005). There is an increased need for biological data of exploited species in order to manage fishing in a more efficient way, either by limiting catches and effort, implementing higher minimum catch sizes or elaborating stocking programs.

On the other hand, in order to facilitate the study of fishery resources in a laboratory, there is a growing need and interest in developing non-invasive technology to monitor marine species for long periods. Telemetry for species is not progressing at the same rate as technology because of the limited market when compared with telemetry for industrial applications (Aguzzi, Company et al., 2011). For that reason, these devices are expensive, do not satisfy some requirements or are not suitable for marine environments (Costa et al., 2012).

To study the behaviour of species in a laboratory, specialized systems are required in order to measure the activity of the individuals as well as to recreate and to simulate the environmental conditions (e.g. light and tidal) that modulate their behaviour. 
In the first place, the measurement of the activity of the species (e.g. detection, location, identification and tracking) can be performed with detectors (Aguzzi, Sarriá et al., 2008), infrared cameras (Sarriá et al., 2008) or Radio Frequency Identification (RFID) technologies (Aguzzi, Sbragaglia et al., 2011).

The second group of required systems are those that recreate the environmental conditions found in real scenarios - the ocean in a laboratory. In this area, (Aguzzi, Sarriá et al., 2008) proposed a scalable hardware architecture for lighting aquariums with blue light (emulating the light spectrum under the sea). In the cited work, the intensity and light cycle control was performed by feeding a set of monochromatic blue LEDs with a pulse-width modulation signal (PWM). Because of its design, the hardware is limited to a power of $2.5 \mathrm{~W}$ (suitable for lighting small aquariums). Moreover, the proposed aerial installation of lights generates reflections affecting the quality of the images (if cameras are used to study the animal behaviour). In this case, image filters or proper camera installation is required.

The water current generation systems for recreating sea currents in aquariums are limited. Currently, the swim tunnels are aquariums with a water current control and are used for studies of physiology, energetics, biomechanics and kinematics of swimming fish (Ohlberger et al., 2007). These systems are small (aquariums no greater than 185 litres) and adapted to study fish (the size and shape of aquarium is unsuitable for behaviour studies of other marine species). On the other hand, circulation pumps for aquariums are controlled by knobs (manual operation) or devices with programmable actions time-triggered but without an interface or open protocol to control them from external applications (Toma et al., 2013). 
This work proposes and evaluates hardware and software solutions to control the lighting and water flow in aquariums suitable for marine species that require higher experimentation areas.

Firstly, the electronics required to control multiple circulation pumps generating at the desired power the water current cycles is outlined.

Secondly, a lighting system design is presented supporting higher power than previous works, allowing the lighting of larger aquariums. The proposed installation and materials used reduces the light reflections and therefore the complexity of camera installation to measure the animal activity from above.

The proposed system has been implemented and evaluated experimentally with the Norway lobster (Nephrops norvegicus L.), decapod that lives in burrows in the muddy bottoms of the shelf and slope of European waters of the Atlantic Ocean and the Mediterranean Sea (Aguzzi and Sardà, 2008). This species uses the burrows which the lobsters themselves dig out as a shelter, and emerge from them according to circadian rhythms, mainly related to light (Sardà and Aguzzi, 2012). However, recent data shows that burrow emergence can be related to other parameters such as the duration and intensity of currents caused by tides or social interactions (Menesatti et al., 2009; Katoh et al., 2013). Identifying and quantifying emergence behaviour related to light and water currents (caused by tides) is the scientific purpose of the system designed.

The system consists of four aquarium tanks divided into three sub aquariums. Each one is equipped with LED lighting and circulation pumps. The system controls the light intensity and cycles, as well as the water flow (speed and duration) of each aquarium. The activity of the species is monitored using video cameras. Machine vision 
algorithms are being developed to identify individuals automatically when they emerge, and to measure the distance travelled outside the burrow (Sbragaglia et al., 2013).

In the next section, the aquariums and the habitat specifications are detailed. Section 3 focuses on the system design, the hydrodynamics and the lighting conditions. The application that manages the system is discussed in section 4 and the materials and methods used in the experiments are detailed in section 5. The results are presented in section 6. Finally, in section 7 the major technological contributions of the developed system are summarized.

\section{Aquariums and specifications habitat}

The system comprises four tanks stacked in two columns (Figure 1). Each aquarium has three sub aquariums (channels) and three burrows (one burrow per channel). The channels have dimensions of $25 \times 53 \times 150 \mathrm{~cm}$.

A mixture of polyester resin and sand has been used to simulate the substrate in the tank and the burrows. The burrows are built with PVC pipes with shape and volume similar to those found in nature (Sbragaglia et al., 2013). Each channel has two openings, through which water is recirculated by a pump (recirculation pipe in Figure 1). Each channel has a strip of LEDs for lighting and an infrared LED strip (used for video recording in darkness).

\section{Control System Design to simulate lighting and hydrodynamics conditions}

The designed system recreates lighting conditions (intensity and duration) and the water currents (speed and duration) at the sea bottom. It has the following characteristics: 
- Water flow control in order to simulate the natural hydrodynamic conditions (e.g. natural currents or tides in different geographic areas).

- Control of intensity and duration of light cycle characteristic photoperiod, to simulate the light in different seasons at different depths, including the gradual increase and decrease of light during the cycles.

Each aquarium channel is equipped with a strip of LED light and a circulation pump. Therefore, the complete system consists of 12 circulation pumps and 12 LED strips. In addition, each channel is equipped with an infrared LED strip to allow the infrared cameras to take photos during the dark phases.

The hardware architecture of the system is shown in Figure 2. It consists of a power supply, circuit breakers, a variable-frequency drive, and a custom electronics with a low-performance processor (PIC16LF88 microcontroller) and a Bluetooth module to receive wirelessly the control commands from an application than runs in a standard computer. Depending on the command received, the microcontroller manages the operation of pumps and lights, regulating the hydrodynamic cycles and the intensity and duration of the photoperiod.

\subsection{Visible LED lighting}

The visible lighting is composed of multiple flexible LED strips (characteristics in Table 1) providing monochromatic blue light. The LED strips were integrated inside semi-submersible methacrylate tubes of $140 \mathrm{~cm}$ length ( 84 LEDs per tube). The power consumption of each lighting tube at full intensity is $6 \mathrm{~W}(12 \mathrm{~V}$ at $0.5 \mathrm{~A})$.

\subsection{Circulation pumps}

The Koralia 7 (Hydor, Italy) were the circulation pumps chosen because their 
specifications ensure good water movement, maintaining low power consumption without causing vibration or noise, appropriate to simulating currents with minimal disturbance to the species. These pumps have an electronic start-up system and a magnetic rotor without axis. The low voltage $\mathrm{AC}$ motor (6 to $14 \mathrm{~V}_{\mathrm{DC}}$ ) of the pump delivers a power between 5 to $19 \mathrm{~W}$, generating flow rates between 900 to 3100 gallons/hour and allowing a water flow between 1 to $10 \mathrm{~cm} / \mathrm{s}$.

\subsection{Infrared LED lighting}

The infrared lighting is independent of the control system and is always on connected directly to a $12 \mathrm{~V}_{\mathrm{DC}}$ power supply. The infrared lighting is based on multiple IR LED strips incorporated into custom semi-submersible methacrylate tubes of $140 \mathrm{~cm}$ length. There is one tube of infrared LEDs installed per channel.

\subsection{Control system}

The regulation of the pumps and lights are performed by the control system, which wirelessly (Bluetooth interface) receives the actions to be applied from an application programmed in LabVIEW and running in a standard computer. The system operates through this application, allowing simple reprogramming of the hydrodynamic and photoperiod cycles required for the biological experiments. In next section, the application and its capabilities are described in greater detail.

The electronic design manages the pumps and lights through a Microchip microcontroller (PIC16LF88), which receives control commands (values of intensity water flow and light) via Bluetooth and acts accordingly increasing/decreasing the water flow of each aquarium and recreating the lighting conditions required - intensity light and time periods for day/night and dawn/dusk. 
The management of the pumps is performed by controlling, from the microcontroller, a variable-frequency drive (VFD). Because of the low power consumption of the selected pumps, only a single VFD is required to handle the twelve pumps. The VFD used is the Dinverter A (Control Techniques, Emerson Industrial Automation- Missouri, USA). This equipment is manual and remote-controllable by commands through a 485 interface. To control this system, the design includes a 485 transceiver to allow the microcontroller interact with the VFD.

VFDs typically have three phase outputs but since the pumps are single phase, only two phases of the VFD are used being wired to a transformer, reducing the output voltage to $12 \mathrm{~V}_{\mathrm{AC}}$ - nominal voltage of the pumps. The transformer output is wired to four circuit breakers (ICP-M 10A de Moeller; Manchester- UK) and four relays that are controllable by the microcontroller (Relay block in Figure 2). The primary is also protected from faults with a two-phase circuit breaker.

The four relays allow the pumps of the four aquariums to be started or stopped independently in a controlled manner. Each output/relay handles the three pumps installed in each aquarium. With this design, it is possible to disable/enable the pumps of each aquarium at any time changing the logic state of the corresponding relay. This feature enables starting the pumps in sequence reducing the current peak required when they are switched on (inductive loads).

The microcontroller proposed had a single USART (Universal Synchronous Asynchronous Receiver Transmitter). In order to handle the communication lines of the VFD and the Bluetooth module, the transmission and reception signals are multiplexed (74HC4051D from NXP) allowing the control of both elements alternatively (Mux block in Figure 2). 
The light control is carried out by PWM. This method is proposed by (Microchip Technology, 2009) and used in previous works for lighting small aquariums with LEDs (Aguzzi, Sarriá et al., 2008). In order to improve the delivered power of the previous works, in this design the LEDs are also controlled by PWM but driven by power MOSFETs.

Each MOSFET drives the lighting of one aquarium but the PWM signal is common for the four gates of the MOSFETs. Therefore, all the aquariums have the same light conditions at the same time.

The light intensity at full power (100\% duty-cycle) can be adjusted inserting the appropriate resistor to the integrated socket in the board design (resistor at the output of the MOSFET and in series with the LEDs). The electronic board design and the lighting system of the four aquariums are powered by a $12 \mathrm{~V}$ power supply (DR model 120-12), able to deliver up to $120 \mathrm{~W}$ (MEAN-WELL, New Taipei City - Taiwan). The $12 \mathrm{~V}$ are also carried to the MOSFETs, providing this working voltage to the LEDs. Each lighting output is protected from short-circuits with a 3 A fuse.

The described system was implemented and mounted in a control cabinet, the distribution of the described components are shown in Figure 3. The four IP67 connectors (I, J, K, L in Figure 3) provide protection from condensation or water contact and contain the wiring of each aquarium. The wiring extends from the four IP67 connectors to four junction boxes installed in the aquariums, where the lights and the pumps are connected. The complete system and the wiring scheme is detailed in Figure 4.

In summary, the control system has the following outputs and features: 
- Four relay controllable outputs with adjustable voltage (6 to $14 \mathrm{~V}_{\mathrm{AC}}$ ) and frequency $(40-63 \mathrm{~Hz})$ to control the circulation pumps. The maximum delivering power is $240 \mathrm{~W}(60 \mathrm{~W}$ per output). Each output feeds the three circulation pumps installed per aquarium. Each output is protected with 10 A circuit breakers.

- Four PWM $12 \mathrm{~V}_{\mathrm{DC}}$ outputs to control the intensity of the visible LED lighting. The maximum current per output is $1.5 \mathrm{~A}$ and a fuse of $3 \mathrm{~A}$ protects each output from short-circuit. The design can handle up to $72 \mathrm{~W}$ (18 W per output). With a resistor, the maximum delivered power per output can be reduced.

\section{System management application}

In this design, although the control system governs the pumps and the lighting, it is an external application developed in LabVIEW for Linux, running in a personal computer, which is responsible for controlling the system by simple orders.

The application developed (Figure 5) automatically sends the appropriate commands to manage the lights and pumps according to the configuration scheduled. The configurator is an event handler, where the intensity power of the pumps and lights are entered together with the execution time (block $\mathrm{C}$ in Figure 5). The actions are triggered by the application comparing the execution time with the system time (computer time clock). If time is exceeded, the associated command to the action is generated and sent wirelessly (Bluetooth) to the control system (microcontroller).

The communication protocol proposed for the system is simple and was programmed previously in the firmware of the microcontroller, in which commands are converted into pump and light actions. In the protocol, there are five possible commands: the intensity power of lights (value of duty-cycle) and pumps (voltage and 
frequency of the VFD), the operation of the relays (open/close) and the operation of the VFD (output on/off). If commands are received and processed correctly by the microcontroller, acknowledgement is sent (echo of the command) from the microcontroller to the computer, so the application knows if the command was successfully executed in the control system.

The light and water current parameters over time are shown in two graphics (A and B blocks in Figure 5) showing the pattern of currents and lights that are going to be recreated in an understandable way.

When the experiment starts by pressing the appropriate button in the application, lights and water currents are recreated according to the configuration scheduled and the system time. The experiment concludes when all the actions scheduled have been executed, but it can be stopped at any time. In both cases the pumps stop automatically, but the lights maintain the last intensity value applied. This allows checking the lights after the experiments for maintenance issues.

The application was installed in a standard personal computer without the LabVIEW Development Environment. So, the application required to run the LabVIEW Run-Time for Linux, which is distributed in rpm packages (Red-Hat distribution architecture supported). These packages were converted to the Debian system architecture using alien application, demonstrating the correct operation of the application in an Ubuntu Operating System.

\section{Validation experiment - Materials and methods}

In order to validate the design and operation of the control system, two types of tests have been carried out. 
In the first set of tests, compliance with the requirements was verified and also the correct electronic operation of the system. The correct response to the commands was also tested and the power of lights and pumps was adjusted.

In the second type of tests whether the system interacted properly in two biological experiments with species was evaluated. This also served to assess the proper operation and robustness of the system working continuously during several weeks. For these experiments, four high definition video cameras with infrared vision capabilities (one camera per aquarium) were installed and used (Figure 4). The cameras were installed to capture the scene of the aquarium from above, and were programmed to automatically shoot photos every ten seconds.

The first biological experiment allowed the correct operation of the lighting system continuously. Individuals ( $N$. norvegicus, $\mathrm{n}=16$ ) were analysed for 10 days simulating lighting conditions in Barcelona (15 h of light, $9 \mathrm{~h}$ of darkness) during the time of the experiment (10 June 2012).

The second experiment was carried out to assess the efficiency of the currents control system. The experiment lasted 10 days during which periodical changes $(12.4 \mathrm{~h})$ in water speed (from 0 to $10 \mathrm{~cm} / \mathrm{s}$ ) with a duration of two hours were applied to simulate a tidal cycle.

The detailed explanation of the experiments and their results are presented in the next section.

\section{Experimental results}

Several tests were performed to validate the correct operation of the system before carrying out the scientific experiments. 
The first set of tests was conducted to validate the correct power management and operation of the system. The energy requirements of the LEDs and pumps were below the specifications limits of the designed system, and both systems operated satisfactorily. However, pumps did not provide full power because a voltage drop of 1 $\mathrm{V}$ due to the cable length in the installation. Two changes were made to solve this power loss; the first was reducing the wiring distance between the pumps and the control system cabinet. The second was adjusting the output voltage of the VDF to provide the working voltage range (characteristics in section 3 ) at the pump terminals.

Specific actions in the firmware of the microcontroller were required in order to control the VFD remotely. First to manage the commands and the protocol of the VFD. Secondly to change the baud-rate of the USART module at runtime (Angulo et al., 2007) depending on whether Bluetooth module or VDF are going to be handled (these devices had different baud-rate).

In a second test, the method to start the twelve pumps was tested. In order to reduce the peak current and the voltage drop (which could cause a bad start or a reverse operation of the pump) when pumps are started, they are switched in sequence through the relays of the proposed design. The test demonstrated the correct operation of the pumps switching each relay ( 3 pumps per relay) every 5 seconds ( 20 seconds to start all the pumps).

After different experimental tests, the pumps were installed at the entrance of the recirculation pipe (pushing water into the pipe). The trials demonstrate improved laminar water current in the aquariums.

The lighting system was evaluated in a third test. Through the microcontroller, the PWM duty-cycle was controlled showing the correct operation of the light 
regulation method. The maximum intensity level was fixed with a $2.2 \mathrm{k} \Omega$ resistor in order to emulate the light intensity of the environment where Norway lobsters are found.

On the other hand, the placement of the lights underwater proved a reduction of reflections in images taken from above with the cameras. Thereby, the installation and materials used were suitable to facilitate appropriate images to the processing stage, an important issue in machine vision systems. In Figure 6 an image acquired during the biological experiments with the lighting system working at full power is shown. Individuals recorded on this image are in two different stages of their typical behaviour: the first, close to the burrow and the second, exploring the surrounding environment.

In the second set of experiments, the interaction of the animals with the programmable stimulus (light and currents) was evaluated along with robustness of the complete system working continuously over several days.

Two consecutive tests were conducted with a total duration of twenty days. The first one allowed the evaluation of light stimulus on animal behaviour. In the second, the interaction of the current with the animals was studied. These experiments demonstrate that the system operates correctly; no anomalies were detected during the twenty days that all the trials lasted.

The activity of the species was measured applying different vision algorithms to the images. The algorithms applied can be found at (Sbragaglia et al., 2013) and produce as output the time series of displacements ( $\mathrm{cm}$ over time) by each individual in the aquarium. The data shown marked emergence rhythms with respect to photoperiod (Figure 7) as well as the current cycle (Figure 8). 
The data analysis proved that individuals have expressed a pattern of emergence related to the darkness phases, with peaks around the simulated sunset as already observed for this species (Aguzzi and Sardà, 2008). It also detects that lobsters usually adopt a freezing behaviour (immobility) when they start to perceive water currents during emergence phases. Moreover, individuals prefer to retreat into the burrow after the onset of water currents. Quantitative and qualitative studies of such behaviours are under investigation at the moment (Sbragaglia et al., Submitted).

\section{Conclusions}

The control system presented in this paper has been successfully tested over several days continuously without failures. The feasibility of controlling the entire aquarium system remotely, via the control system implemented, has been a significant advantage in this type of laboratory experiments where the presence of any kind of disturbance (students, light contamination, noise, vibration) may compromise the results interfering with the behavioural output under study.

The ability to remotely control the system was achieved by using the Bluetooth interface, which avoids the physical connection with the computer and the additional design of a protection optocoupler interface. On the other hand, the low cost of a Bluetooth OEM module, represents, in this area, an interesting alternative compared to conventional wiring designs, where the cost of cable, connectors, protection elements, and the installation in the aquarium environment, could exceed the costs of these type of Bluetooth modules.

The implemented system used commercial equipment (pumps and lights) and a custom board design to control all the elements. The system has also shown 
considerable ease of use through the application developed, allowing the experiments were conducted easily.

The programmable light and current cycles allowed the study of Nephrops norvegicus behavioural rhythms presenting a great innovation with respect to previous devices: the possibility to simulate a natural scenario where both light and water currents interact to shape the habitat of the species in several aquariums at the same time. Water currents are known to interfere with the lobster's behaviour (Newland et al. 1988) but the way in which periodical water currents affect the behavioural rhythms of the species was still missing information with an intrinsic value for species management. Moreover, the possibility to use video image analysis permitted performing qualitative study on the response of the lobsters to water currents.

The system presented high potentiality for future experiments to address questions regarding the ecology and biology of the species with particular focus on the importance of Nephrops for European fishery and deep water marine chronobiology.

\section{Acknowledgments}

The authors would like to acknowledge the financial support of "Ministerio de Ciencia e Innovación" in the grant "Ritmos fisiológicos e influencia del hábitat en crustáceos marinos profundos" (RITFIM; CTM2010-16274 CICYT). PI: Dr. J. Aguzzi

\section{References}

Aguzzi, J., Sardà, F., "A history of recent advancements on Nephrops norvegicus behavioral and physiological rhythms", Rev. Fish Biol. Fisheries 18, 235-248, 2008

Aguzzi, J., Sarriá, D., García, J. A., del Río, J., Sardà, F., Mànuel, A. "A new tracking system for the measurement of diel locomotor rhythms in the Norway lobster, Nephrops norvegicus (L.)". Journal of Neuroscience Methods, 173(2), 215-224, 2008 
Aguzzi, J., Company, J.B., Costa, C., Menesatti, P., García, J.A., Bahamon, N., Puig, P., Sardà, F. "Activity rhythms in the deep-sea crustacean: chronobiological challenges and potential technological scenarios", Frontiers in BioscienceLandmark 16: 131-150, 2011

Aguzzi, J.; Sbragaglia, V.; Sarriá, D.; García, J.A.; Costa, C.; del Río, J.; Mànuel, A.; Menestti, P.; Sardà, F.; “A new RFID technology for the laboratory monitoring of behaviour in marine organisms", Sensors-Basel (Instruments and Instrumentation) 11: 932-9548, 2011

Angulo, J. M., Angulo, I., Extebarría, A., "Microcontroladores PIC - Diseño práctico de aplicaciones, $1^{\text {a }}$ parte, $4^{\mathrm{a}}$ edición”, Editorial McGraw-Hill, ISBN 8448156471, 2007

Costa, C., Antonucci, F., Pallottino, F., Aguzzi, J., Sarriá, D., Menesatti, P. “A review on agri-food supply chain traceability by means of RFID technology", Food and Bioprocess Technology In Press, 2012

Csirke, J. "Global production and state of marine fishery resources", FAO Marine Resources Service, Fishery Resources Division, Review of the state of world marine fishery resources, FAO Fisheries Technical Paper. No.457, Rome, 2005

Katoh, E., V. Sbragaglia V., Aguzzi, J., Breithaupt, T. "Sensory biology and behaviour of Nephrops norvegicus." The ecology and biology of Nephrops norvegicus. Adv. Mar. Biol 64: 65-106, 2013

Menesatti, P., Aguzzi J., Costa, C., García, J.A., Sardà, F. "Video-image analysis for microcosm experiments on activity rhythms with multiple individuals of Norway lobster, Nephrops norvegicus (L.)", Journal of Neuroscience Methods 184: 161-168, 2009

Microchip Technology, "Compiled Tip 'N Tricks Guide”, Datasheet DS01146B, Microchip Technology, 2009

Newland, P., D. Neil and C. Chapman, "The reactions of the Norway lobster, Nephrops norvegicus (L.), to water currents." Marine \& Freshwater Behaviour \& Phy 13(3): 301-313, 1988

Ohlberger, J., Staaks, G., Hölker, F. "Effects of temperature, swimming speed and body mass on standard and active metabolic rate in vendace (Coregonus albula)". Journal of Comparative Physiology. B, Biochemical, Systemic, and Environmental Physiology, 177(8), 905-16, 2007 
Sardà, F., Aguzzi, J., "A review of burrow counting as an alternative to other typical methods of assessment of Norway lobster populations", Reviews of Fish Biology and Fisheries (Fisheries) 22: 409-422, 2012

Sarriá, D., del Río, J., Mànuel, A., Aguzzi, J., García, J. A., Sardà, F. "Infrared and Imaging Application to measure emergence activity rhythms on Nephrops norvegicus (L.) population Assessment". In IEEE Sensors Applications Symposium (pp. 74-78. ISBN 978-1-42441963-0). Atlanta, USA, 2008

Sbragaglia, V., Aguzzi, J., García, J., Sarriá, D., Gomáriz, S., Costa, C., Menesatti, P., Vilaró, M., Manuel, A., Sardà, F. "An automated multi-flume actograph for the study of behavioral rhythms of burrowing organisms." Journal of Experimental Marine Biology and Ecology 446: 177-185. 2013

Sbragaglia, V., Aguzzi, J., Chiesa, J. J., García, J. A. The effect of periodical water currents on the burrow emergence rhythm of the Norway lobster (Nephrops norvegicus, L.). submitted.

Toma, D. M., Bresco, D. P., Mànuel, A., Masalles, J. M. "An impacting energy harvester through piezoelectric device for oscillating water flow". In 5th Martech International Workshop on Marine Technology (pp. 39-42). ISBN 97884-616-5764-3, 2013 
Table 1 Visible LED lighting characteristics

\begin{tabular}{|c|c|}
\hline Light Colour & Blue $472 \mathrm{~nm}$ \\
\hline Beam Angle & $120^{\circ}$ \\
\hline Power & $4.8 \mathrm{~W} / \mathrm{m} 1.5 \mathrm{~W} / \mathrm{ft}$. \\
\hline Input Voltage & $12 \mathrm{~V}_{\mathrm{DC}}$ \\
\hline Number of LEDs & 60 per meter \\
\hline Minimum cutting increment & $50 \mathrm{~mm}, 1.97 \mathrm{in}$ \\
\hline Width and Height & $8 \mathrm{~mm}, 0.31 \mathrm{in} / 2 \mathrm{~mm}, 0.08$ in \\
\hline Average Lifetime & 50.000 hours \\
\hline
\end{tabular}

Figure 1 Diagram of Aquarium (adapted from Sbragaglia et al., 2013)

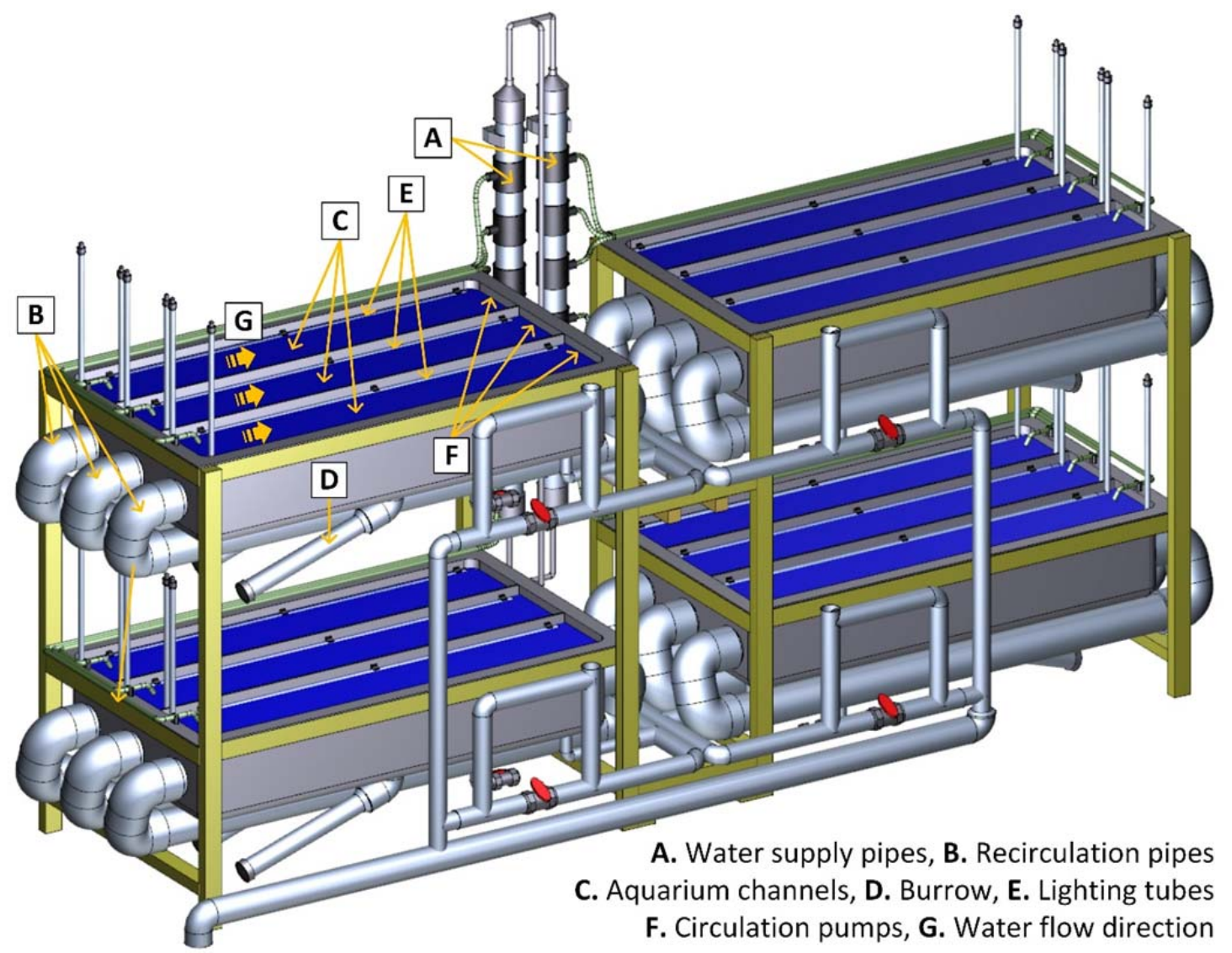


Figure 2 Block diagram of the developed electronic control system

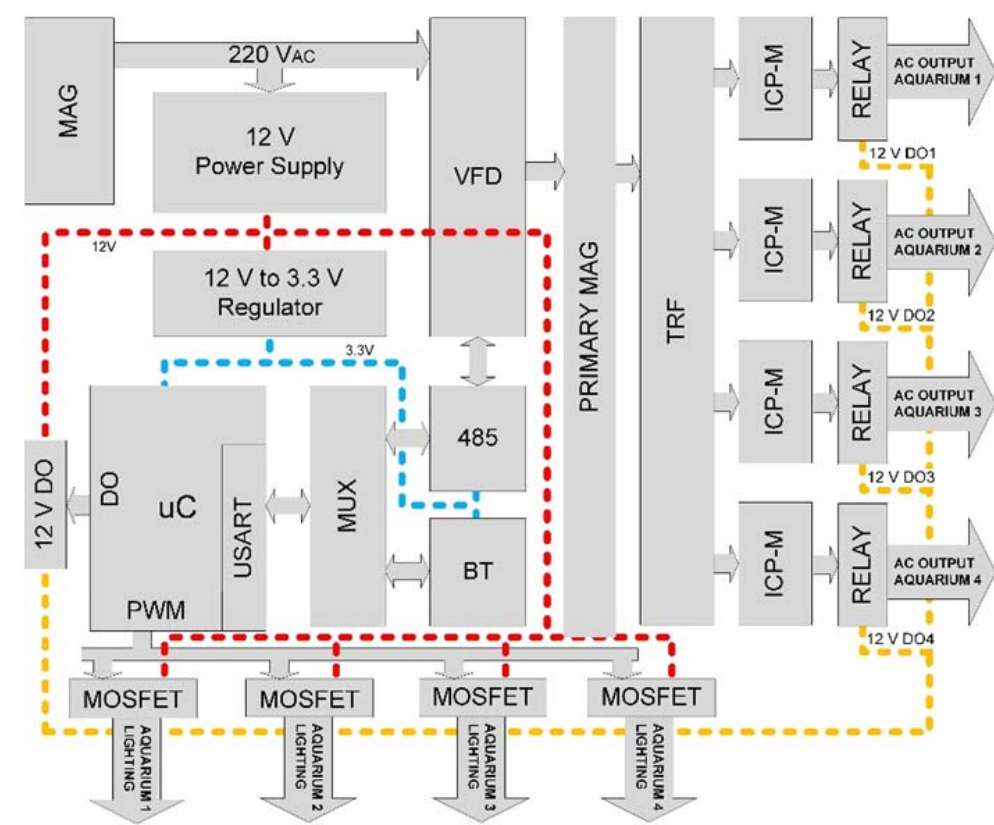

Figure 3 Elements of the control system in the cabinet

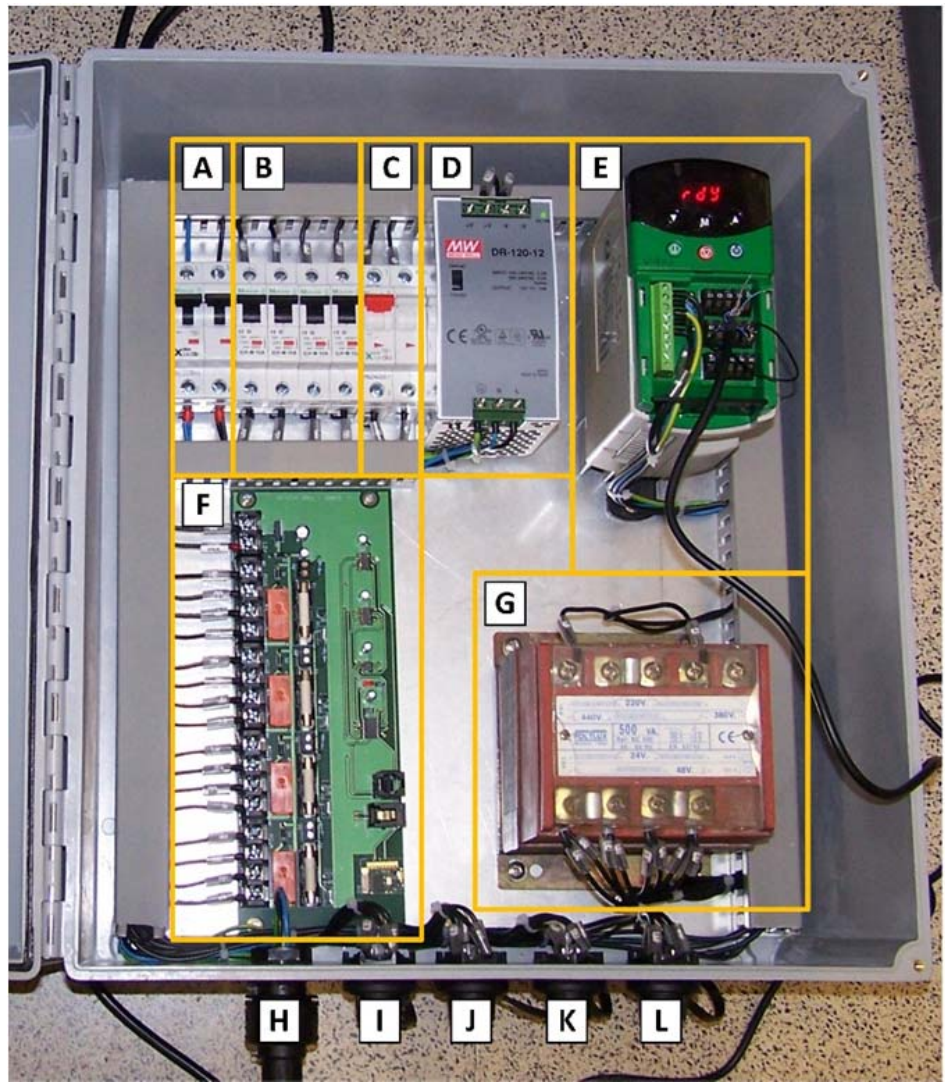

MAG. Main circuit breaker ICP-M. 10 A circuit breaker for the AC output (circulation pumps) uC. Microcontroller DO. uC digital outputs $12 \mathrm{~V}$ DO. Driver for digital outputs USART. the USART module of uC MUX. Multiplexor

PWM. The PWM module of $U C$ BT. Bluetooh module 485. 485 to USART transceiver VFD. Variable Frequency Drive PRIMARY MAG. Primary circuit breaker

TRT. Transformer RELAY. Relays controlling the operations of pumps MOSFET: Power Mosfet to feed the lighting system
A. Main circuit breaker
B. Breakers for AC outputs, the circulation pumps
C. Primary circuit breaker
D. $12 \mathrm{~V}$ power supply
E. Variable Frequency Drive
F. PCB Board design
G. Transformer
H. $220 \mathrm{~V}$ mains
I. Output for aquarium 1
J. Output for aquarium 2
K. Output for aquarium 3
L. Output for aquarium 4 
Figure 4 Installation diagram

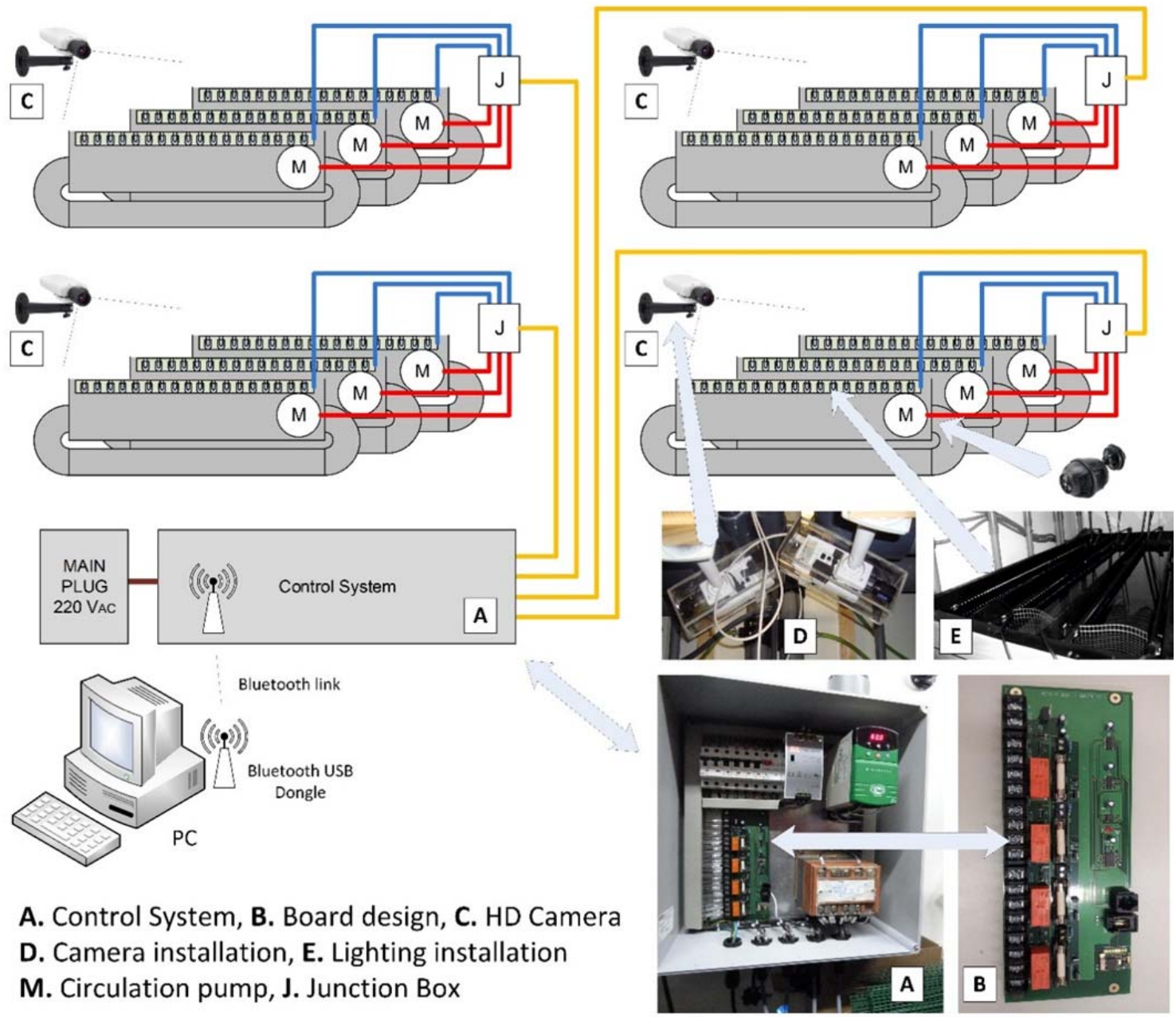


Figure 5 Front panel of the application

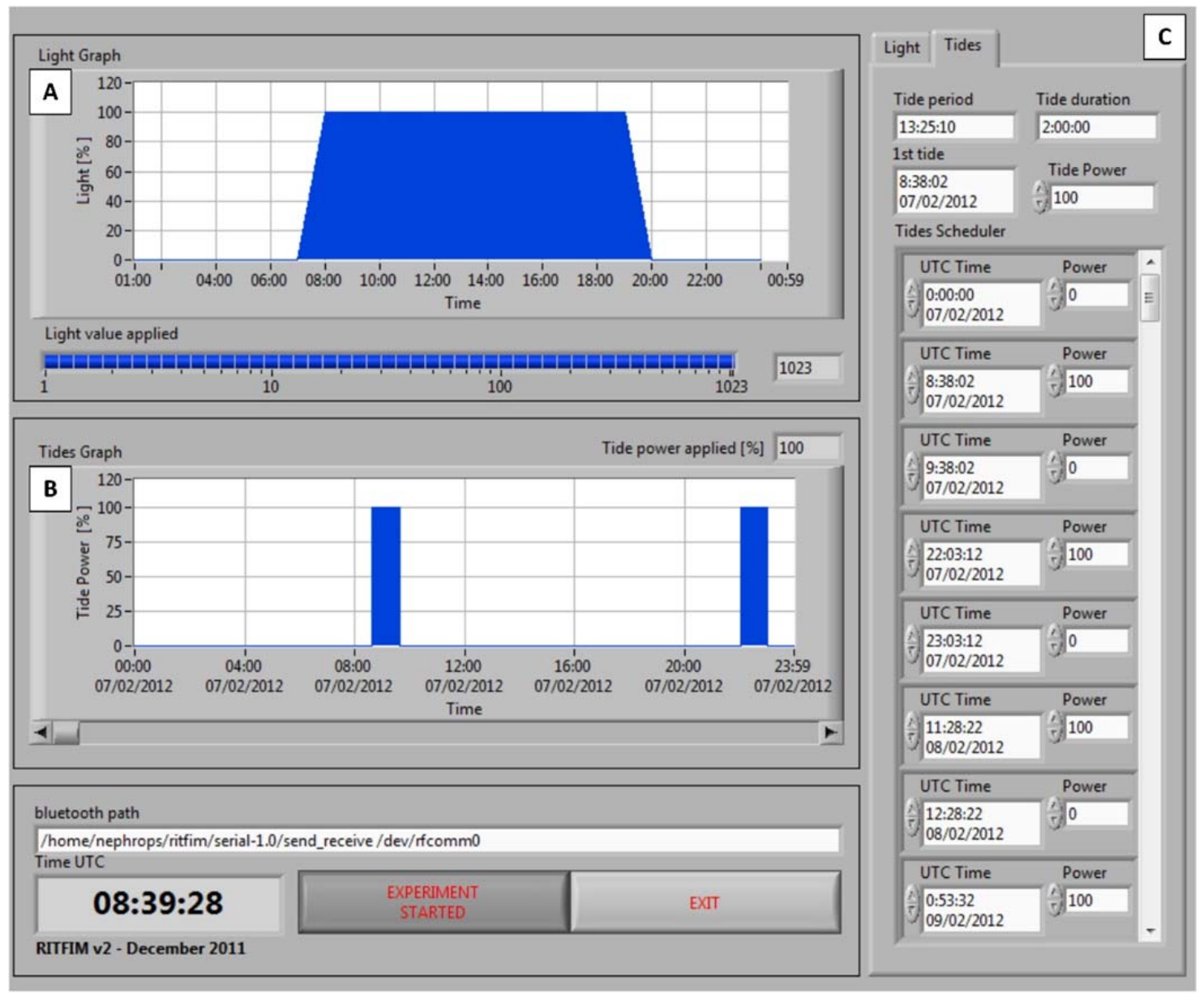

A. Light settings, B. Water current settings, C. Light and water current configurator 
Figure 6 Image acquired during the experiments

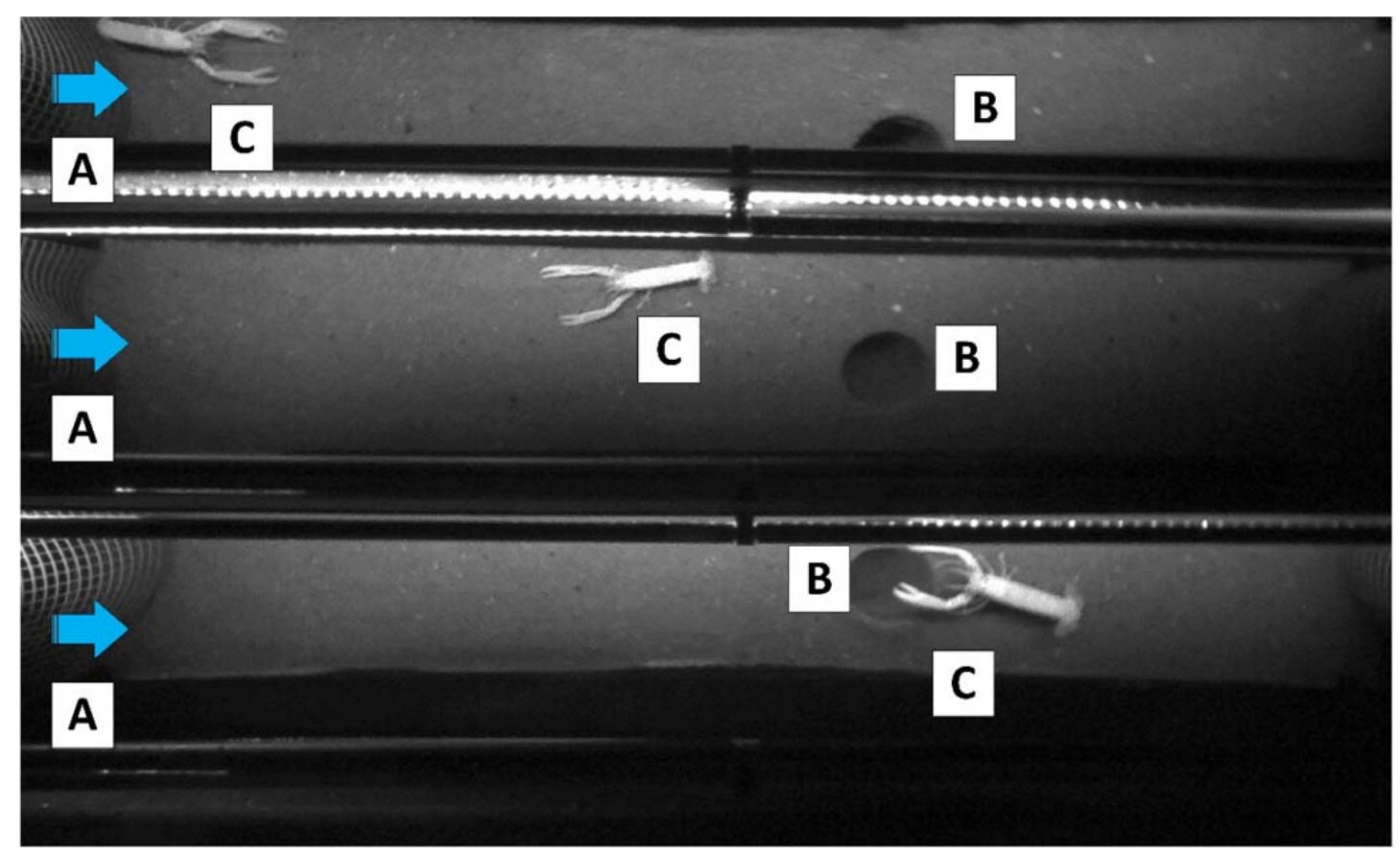

A. Water flow direction, B. Burrow, C. Individuals in the experiment 
Figure 7 Time series displacement of an individual during the 1st experiment. The black lines represent the number of centimetres travelled by the individual during 10 days. The grey curves represent the light cycle applied (15-9 LD) and managed by the control system described in this work

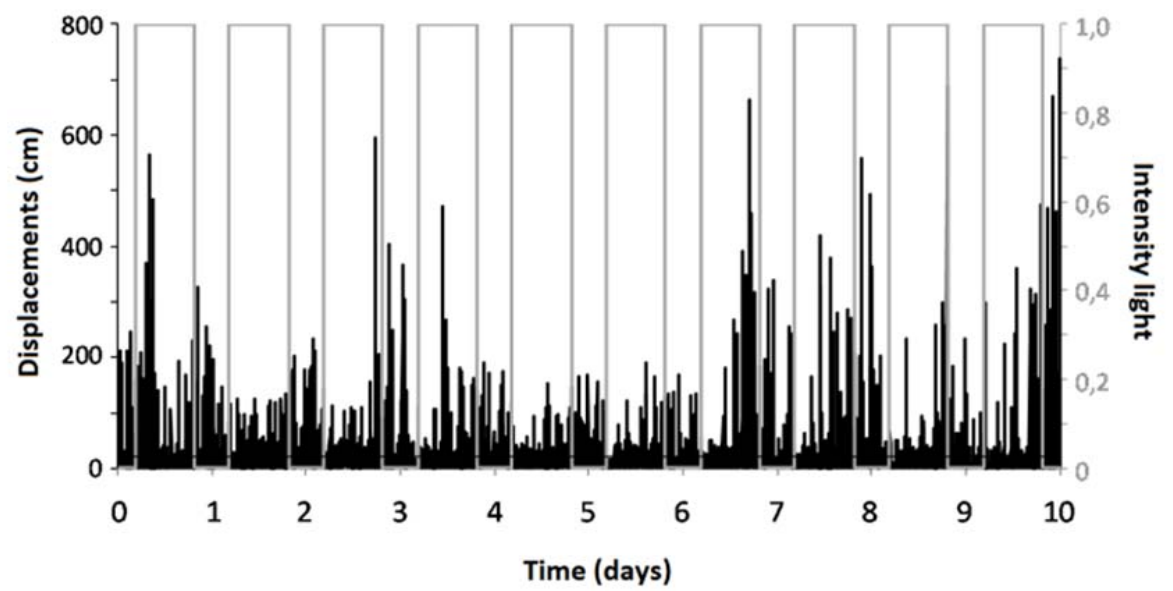

Figure 8 Time series displacement of an individual during the 2nd experiment. The black lines represent the number of centimetres travelled by the individual during 10 days. The grey curves represent the water current cycles applied (periodicity of $12.4 \mathrm{~h}$ ) and managed by the control system described in this work

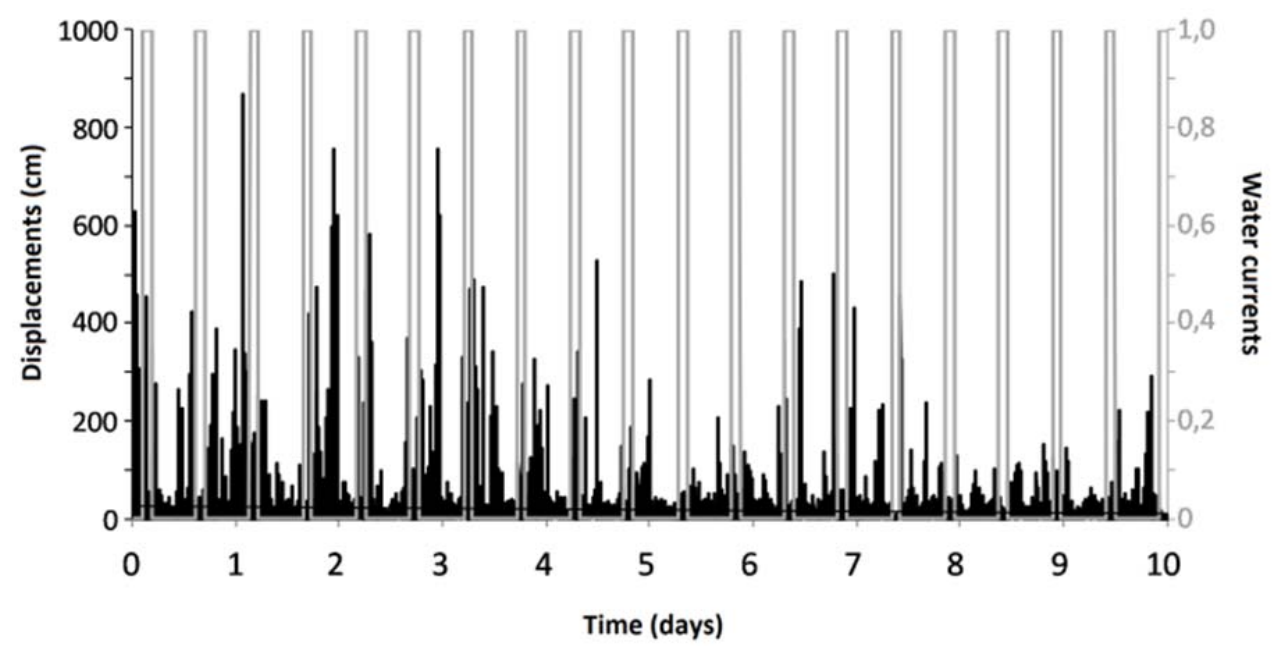

